\title{
Dalbavancin Efficacy and Impact on Hospital Length-of-Stay and Treatment Costs in Different Gram-Positive Bacterial Infections
}

\author{
Mariacristina Poliseno ${ }^{1,9}$ - Davide Fiore Bavaro ${ }^{1} \cdot$ Gaetano Brindicci $^{1} \cdot$ Giovanni Luzzi $^{2} \cdot$ Domenico Maria Carretta $^{3}$. \\ Antonio Spinarelli ${ }^{4} \cdot$ Raffaella Messina $^{5} \cdot$ Maria Paola Miolla $^{4} \cdot$ Teresa Immacolata Achille $^{2}$. \\ Maria Rosaria Dibartolomeo ${ }^{6}$. Maria Dell'Aera ${ }^{6}$. Annalisa Saracino ${ }^{1}$. Gioacchino Angarano ${ }^{1} \cdot$ Stefano Favale ${ }^{2}$. \\ Carlo D'Agostino ${ }^{3} \cdot$ Biagio Moretti ${ }^{4} \cdot$ Francesco Signorelli $^{5} \cdot$ Camilla Taglietti $^{7} \cdot$ Sergio Carbonara ${ }^{1,8}$
}

Accepted: 19 March 2021 / Published online: 21 April 2021

(c) The Author(s) 2021

\begin{abstract}
Background and Objectives The study aimed to evaluate the impact of dalbavancin therapy on both hospital length-of-stay (LOS) and treatment-related costs, as well as to describe the clinical outcome, in a retrospective cohort of patients with diverse Gram-positive bacterial infections, hospitalized in different specialty Units.

Methods From July 2017 to July 2019, clinical and sociodemographic data were collected for all hospitalized patients switched to dalbavancin for the treatment of Gram-positive infections. LOS and treatment-related costs were assessed and compared to a hypothetical scenario where the initial standard antimicrobial therapy would have been administered in hospital for the same duration as dalbavancin.

Results A total of 50 patients were enrolled. The observed infections were: acute bacterial skin and skin structure infections (ABSSSIs, 12 patients), complicated ABSSSIs (eight patients), osteoarticular infections (18 patients), vascular graft or cardiovascular implantable electronic devices (CIED) infections (12 patients). After a median of 14 [interquartile range (IQR) 7-28] days, the in-hospital antimicrobial therapy was switched to dalbavancin $1500 \mathrm{mg}$. When appropriate, considering the site and the clinical course of the infection, $1500 \mathrm{mg}$ doses were repeated every 14 days until recovery. Overall, 49/50 (98\%) patients reported clinical success at the end of therapy. No relapses were observed in 37 patients for whom a median follow-up of 150 (IQR 30-180) days was available. By switching to dalbavancin, a median of $€ 8,259$ (IQR 5644-17,270) and 14 hospital days (IQR 22-47) per patient were saved.

Conclusions In this experience, the use of dalbavancin contributed to shorten LOS and treatment-related costs, especially in difficult Gram-positive infections requiring prolonged therapy.
\end{abstract}

\section{Introduction}

Gram-positive bacteria represent a frequent, well-recognized cause of hospital- and community-acquired infections in Europe, with an estimated expense for European healthcare systems of $€ 380$ million only for methicillin-resistant Staphylococcus aureus (MRSA) infections, which affect about 150,000 people per year [1].

Despite acute bacterial skin and skin structure infections (ABSSSIs) being the most common Gram-positive-caused infectious diseases, the major burden in terms of morbidity, mortality, prolonged hospitalizations, and costs is sustained

Mariacristina Poliseno

polisenomc@gmail.com

Extended author information available on the last page of the article

\section{Key Points}

The switch to dalbavancin in patients hospitalized for diverse Gram-positive infections allowed a significant reduction both in the duration of hospital stay and in treatment-related costs, along with an overall good clinical outcome.

These benefits were greater for complex infections requiring prolonged antimicrobial treatment.

If confirmed by larger studies, this therapeutic approach could have a remarkable impact on the management of infections requiring prolonged antimicrobial treatment. 
by Gram-positive-related cardiovascular and osteoarticular infections, especially when implants are involved $[2,3]$.

Stepping down from intravenous to oral treatment is one of the major challenges in these clinical situations, since this process is frequently hindered by pharmacokinetic issues, by the need for drugs with anti-biofilm activity, or by the presence of multidrug-resistant bacteria.

In these cases, outpatient parenteral antimicrobial therapy (OPAT) could allow daily use of hospital-restricted, intravenous antimicrobials, but, mainly because of organizational or financial inadequacies, this option is often not very accessible in many geographic areas in Italy, as well as elsewhere. As a consequence, in these areas, prolonged hospitalization is frequently required as the only way to guarantee these therapies, with a consequent huge organizational and financial burden for the National Healthcare System, as well as associated discomfort and risk for hospital-associated complications for the patient $[3,4]$.

Moreover, a daily intravenous therapy, delivered either in hospital or in an outpatient setting, implies additional issues, including the need for a long-lasting intravenous (IV) line, which is also associated with patient discomfort, reduced quality of life, and the risk of several local and systemic complications (e.g., thrombophlebitis, cellulitis, bacteremia, endocarditis, sepsis) $[5,6]$.

The introduction of long-acting IV antimicrobials has represented a turning point in recent years: among them, dalbavancin is a second-generation, semi-synthetic intravenous antibiotic, with high efficacy in most Gram-positive bacteria, including multidrug-resistant strains [6].

The high bactericidal and anti-biofilm activity $[7,8]$, as well as the favorable safety and tolerability profile $[9,10]$, could allow dalbavancin to be used in a wide range of difficult Gram-positive infections beyond ABSSSIs, which is its only current authorized indication both in the USA and the EU [6, 10-12]. In addition, the long-lasting activity of dalbavancin (at least 14 days with a single $1500 \mathrm{mg}$ dose [13]) makes this antibiotic especially suitable for treating infections in an outpatient setting, including those needing prolonged therapies.

As a consequence, the use of dalbavancin might help to reduce both the hospital length-of-stay (LOS) and treatmentrelated costs, avoid the need for a mid-term intravenous line, and increase patient compliance, at least in subjects not eligible for either oral regimens or daily outpatient parenteral antibiotic therapy (OPAT).

Studies published thus far have investigated the efficacy, safety, and tolerability of dalbavancin [14, 15], but only a few of them have attempted to assess its impact on hospital LOS and treatment-related costs, mainly with the following approaches: (a) systematic reviews, network meta-analysis, and cost analysis of data from the current literature [16]; (b) description of therapeutic strategies performed in real-life settings that have included dalbavancin and estimation of the related economic advantages/disadvantages [17-19]; (c) expense simulation models based on data collected in a real-life scenario, performed with a mono- or multi-centric design [20, 21].

Although providing remarkable insights, these studies were still affected by different limitations: some of them were based on simulation models rather than on a real-life approach [20, 21]; others considered ABSSSI as the sole infection studied [16-18]; in some researchs, patient enrollment was performed from a single specialty Unit $[18,21]$; in other reports, cost analysis was based on a generic estimated, rather than individually calculated, cost of standard therapy in medical units [19-21].

The objective of this study was to evaluate the extent to which the switch from a standard intravenous antimicrobial therapy to dalbavancin could impact both the hospital LOS and treatment-related costs in diverse Gram-positive bacterial infections, including implant-associated and other difficult-to-treat infections. Clinical outcomes were also described.

\section{Methods}

We retrospectively enrolled all patients consecutively hospitalized in speciality units of the University Hospital Policlinico of Bari from 1 July 2017 to 1 July 2019 who met all the following inclusion criteria: (a) age $\geq 18$ years; (b) definite or highly suspected diagnosis of Gram-positive bacterial infection; (c) initiation of a daily intravenous therapy with standard antibiotics registered in Italy for hospital use only (e.g., daptomycin, vancomycin, teicoplanin $400 \mathrm{mg} / \mathrm{vial}$ ), which was subsequently switched to a dalbavancin regimen prescribed by an infectious diseases (I.D.) consultant.

Given the retrospective, real-life approach of the study, antimicrobial treatment schedules, including the switch to dalbavancin therapy and its duration, were prescribed exclusively based on the clinical judgment of the I.D. consultant, taking into consideration the site and severity of the infection, the isolated pathogens and their drug susceptibility, as well as the individual patient clinical course, independently of the purpose of this study.

Demographic data, medical history, infection site, presenting clinical and laboratory characteristics, clinically significant microbial isolates and their drug-susceptibility, antibiotic treatment (molecules, dose, and duration), and clinical outcome both at the end of treatment (EOT, as defined below) and at follow-up visits were retrieved for each patient from medical charts. Reasons behind the switch to dalbavancin and antimicrobial-related adverse effects were also recorded. 
In case of treatment success (see definition below) at the EOT, follow-up visits were recorded in order to diagnose any relapse of the treated infection. In case of death, the cause and degree of correlation with the infection were assessed.

\subsection{Study Design and Definitions}

Figure 1 illustrates the study design. Firstly, data regarding timing of hospital admission and discharge, type and duration of both the initial in-hospital standard antimicrobial therapy and dalbavancin treatment were collected.

The following variables were assessed:

- End of treatment (EOT) was defined as the 14th day after a single $1500 \mathrm{mg}$ dose of dalbavancin, or, for longer treatments with repeated infusions of $1500 \mathrm{mg}$ at 14-day intervals, as the 14 th day after the last dose. This definition was based on the minimum duration of effective serum dalbavancin concentrations reported in the literature, following the doses specified above [6].

- Success was defined as stable remission, detected at the EOT, of both symptoms and signs of infection recorded at patient presentation, along with a stable reduction of both C-reactive protein (CRP) to a value $<10 \mathrm{mg} / \mathrm{L}$ and procalcitonin $(\mathrm{PCT})$ to $<0.5 \mathrm{ng} / \mathrm{mL}$, if not altered for other reasons than the infection. Furthermore, follow-up cultures, when indicated either from blood or from other sites of infections, had to be negative.

- Failure was defined as either the persistent alteration at the EOT of the infection-related, clinical, and laboratory findings specified above, or the positivity of follow-up cultures.

- Duration of dalbavancin treatment was the number of days from the switch to dalbavancin treatment to the EOT, as defined above.

- Actual hospital length-of-stay was defined as the duration of in-hospital stay from the day of admission to the day of discharge.

The expense incurred in the actual scenario was calculated for the study population, both for hospital accommodation and for dalbavancin treatment, according to the following definitions:

- Daily cost of hospital accommodation was the daily cost of each bed place with respect to both the patient stay and meals, excluding the costs of any diagnostic and treatment interventions, as based on the annual expense report (year 2018) of each participating hospital unit. Table 1 reports the calculation model of this variable for the Unit of Infectious Diseases as an example.

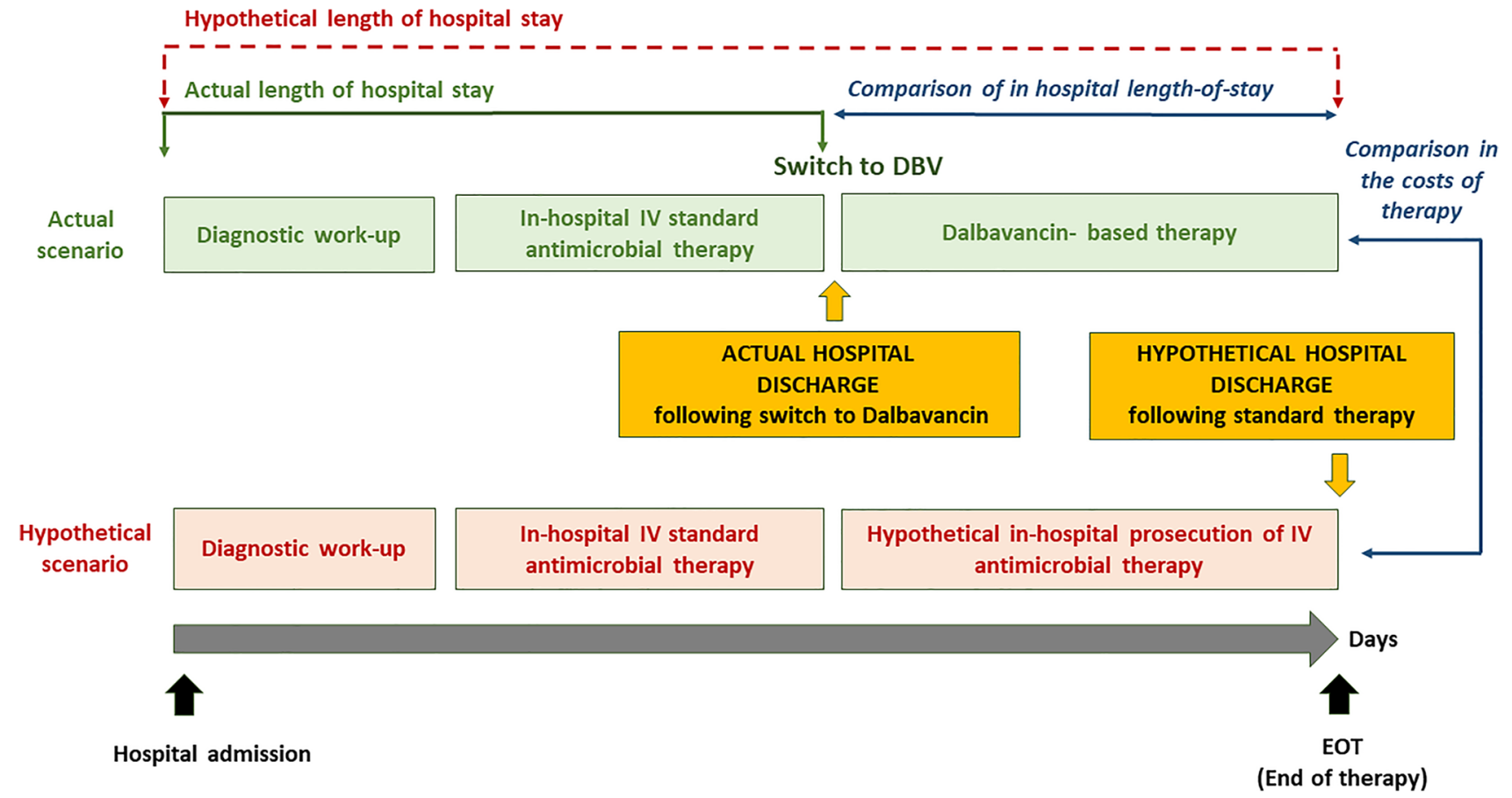

Fig. 1 Study design. Intra-patient comparison of hospital length-ofstay and costs of treatment between actual scenario (switch of in-hospital antimicrobial therapy to dalbavancin) and hypothetical scenario (no switch to dalbavancin, continuation of in-hospital standard antimicrobial therapy for the same number of days-see text for definitions) 
- Cost of actual dalbavancin treatment was calculated for each patient using the following formula (tax-free prices were considered):

[(price of each dalbavancin vial $\times$ total number of vials infused $)+$ (price of intravenous infusion sets and all other accessories* used for one dose infusion $\times$ number of doses $)+$ (daily cost of hospital accommodation $\times$ number of days of hospital-stay after the switch to dalbavancin)]

* Infusion accessories: needles, gloves, antiseptic, gauze, plasters.

Finally, both the actual hospital length-of-stay and the cost of actual dalbavancin treatment were compared for each patient to a hypothetical, intrapatient control scenario where no switch to dalbavancin treatment would have occurred and, consequently, the previous intravenous, standard antimicrobial therapy would have been prolonged in hospital for the

Table 1 Model for the calculation of the daily cost of hospital accommodation for one bed place-Infectious Diseases and Tropical Medicine Unit-Inpatient Ward (year 2018)

\begin{tabular}{|c|c|}
\hline Variables & Values \\
\hline \multicolumn{2}{|l|}{ Direct costs $(€)$} \\
\hline Non-health-care-related consumer goods ${ }^{\mathrm{a}}$ & 6117 \\
\hline Personnel costs & $2,274,308$ \\
\hline Health-care services and management-related costs ${ }^{\mathrm{b}}$ & 380,761 \\
\hline Depreciations and provisions & 656 \\
\hline Total & $2,661,842$ \\
\hline \multicolumn{2}{|l|}{ Indirect costs $(€)$} \\
\hline Department common costs ${ }^{\mathrm{c}}$ & 47,013 \\
\hline Hospital common costs ${ }^{\mathrm{d}}$ & 198,067 \\
\hline Total & 237,244 \\
\hline $\begin{array}{l}\text { Total Direct + Indirect costs for hospital accommodation } \\
(€)\end{array}$ & $2,899,085$ \\
\hline Inpatients bed places (no.) & 22 \\
\hline $\begin{array}{l}\text { Unit patient-days capacity of hospitalization per year } \\
\text { (no.) }\end{array}$ & 7300 \\
\hline $\begin{array}{l}\text { Daily cost of hospital accommodation for one bed place } \\
(€)^{\mathrm{e}}\end{array}$ & 361 \\
\hline
\end{tabular}

${ }^{a}$ Consumer goods including food, laundry, and cleaning materials, printers and computers with related IT-services, maintenance services, and others

${ }^{\mathrm{b}}$ Including concierge, cleaning, laundry, and catering services, bursar office, collection and transportation of hazardous and non-hazardous special waste, HVAC (heating, ventilation and air conditioning) service, general maintenance, leasing rental and other financial services

${ }^{c}$ Pharmacy, warehouse, others

${ }^{\mathrm{d}}$ Directorate, general offices, staff offices, management areas, and others

${ }^{\text {e}}$ Daily cost of hospital accommodation: [(Total Direct + Indirect costs for hospital accommodation)/Unit patient-days capacity of hospitalization per year)] same duration as the actual dalbavancin therapy. We chose this hypothetical control scenario because patients were not eligible for oral treatment and, as mentioned in Introduction section, an adequate OPAT program is not yet properly organized and accessible in a timely manner in our geographic area, as well as elsewhere.

Furthermore, we assumed that standard antimicrobial therapy would have been prescribed at least for the same duration as dalbavancin, based both on published evidence on dalbavancin's non inferiority with respect of common antimicrobials requiring daily infusion [13] and on the awareness that the I.D. consultant did usually prescribe dalbavancin for the same treatment duration recommended for standard antimicrobials according to the infection site. Finally, we assumed as well that individual patient factors (e.g., initial clinical severity, immune depression, complications) that in some cases required a longer therapy duration than recommended would have likely received standard antimicrobials for an equivalent duration.

Possible biases and limitations associated with the study design illustrated here, and chosen by others as well [20-22], are addressed in the Discussion (section 4.1) of the article.

In this hypothetical control scenario, we assessed the following variables:

- Hypothetical hospital LOS was meant as the number of days of hospital-stay starting from admission until the actual dalbavancin EOT, as defined above.

- Daily cost of standard antimicrobial therapy referred to expense for 1 day of therapy with standard antimicrobial treatment and was calculated as follows (tax-free prices were considered for each item):

[(price of each antimicrobial vial $\times$ number of vials infused per day) + (price of intravenous infusion sets and all other accessories* used for one day of therapy) + cost of therapeutic drug monitoring tests, when performed (i.e., for vancomycin, teicoplanin, aminoglycosides)]

* Infusion accessories: needles, gloves, antiseptic, gauze, plasters.

Assuming that the cost of routine blood tests is comparable among different antimicrobial therapies, including with dalbavancin, this expense was not calculated.

- Cost of hypothetical continuation of in-hospital standard treatment was calculated as follows: [(daily cost of standard antimicrobial therapy $x$ duration of dalbavancin treatment $)+$ (daily cost of hospital accommodation $x$ duration of dalbavancin treatment)]. 


\subsection{Statistics}

Descriptive statistics were produced for demographic, clinical, and laboratory characteristics of cases. Mean and standard deviation (SD) are presented for normally distributed variables, median and interquartile range (IQR) for nonnormally distributed variables, number and percentages for categorical variables. Groups were compared with parametric or nonparametric tests, according to data distribution, for continuous variables, and with Pearson's $\chi^{2}$ test (Fisher's exact test where appropriate) for categorical variables.

\section{Results}

\subsection{Clinical Features of the Study Population}

Fifty patients met the study inclusion criteria. These subjects were hospitalized in the following units: Infectious Diseases, Rheumatology, Cardiology (two different units), Neurosurgery, Urology, Nephrology, Orthopedics.

Characteristics of the study population are reported in Table 2. Patients were mainly males (34 patients 68\%), median age was 61 (IQR 61-75) years; at least one comorbidity was detected in $80 \%$ of cases (median Charlson Comorbidity Index =3, IQR 1-4).

Based on the site of infection, enrolled patients were divided into the following four groups:

- $\operatorname{ABSSSIs}(n=12$ patients, $24 \%$ ), diagnosed according to the US Food and Drug Administration (FDA) definition [22], mainly limbs or facial cellulitis (86\%);

- Complicated ABSSSI ( $n=8$ patients, $16 \%)$ : patients who presented with an ABSSSI but in whom several clinical complications were subsequently diagnosed and which impaired the clinical course, i.e., bone (50\%) or joint $(12.5 \%)$ involvement, recurrent erysipelas (25\%), pelvic abscess with pelvic inflammatory disease $(12.5 \%)$;

- Bone and joint infections $(n=18$ patients, $36 \%)$, including native vertebral infections (44\%), long-bone osteomyelitis (25\%), prosthetic joint infections (12.5\%), septic arthritis (19\%);

- Cardiovascular implant infections $(n=12$ patients, $24 \%)$, 11 of which involved a cardiac implantable electronic device (CIED) infection and one case was an aortic vascular graft infection.

Table 2 Clinical and microbiological features of the study population at presentation

\begin{tabular}{|c|c|c|c|c|c|}
\hline Variables & Total $(n=50)$ & ABSSSIs $(n=12)$ & $\begin{array}{l}\text { Complicated } \\
\text { ABSSSIs } \\
(n=8)\end{array}$ & $\begin{array}{l}\text { Bone } \\
\text { and joint } \\
\text { infections }^{\mathrm{a}} \\
(n=18)\end{array}$ & $\begin{array}{l}\text { CIED/vascular grafts } \\
\text { infections }^{\mathrm{b}}(n=12)\end{array}$ \\
\hline Age, years, median (IQR) & $61(51-75)$ & $59(47-76)$ & $56(50-77)$ & $55(48-75)$ & $66(58-71)$ \\
\hline Male sex, $n(\%)$ & $34(68)$ & $6(50)$ & $3(37)$ & $14(77)$ & $11(91)$ \\
\hline$\geq 1$ co-morbidity, $n(\%)$ & $41(82)$ & $10(83)$ & $8(87)$ & $12(66)$ & $11(91)$ \\
\hline Cardiocirculatory disease & $27(54)$ & $7(58)$ & $5(62)$ & $4(22)$ & $11(91)$ \\
\hline COPD & $3(6)$ & $1(8)$ & $1(12)$ & $1(6)$ & - \\
\hline Renal impairment & $3(6)$ & $1(8)$ & - & $2(11)$ & - \\
\hline Solid tumours & $4(8)$ & - & $1(12)$ & $3(16)$ & - \\
\hline Others & $13(7)$ & $1(8)$ & $2(1)$ & $5(27)$ & $5(27)$ \\
\hline Charlson Comorbidity Index, median (IQR) & $3(1-4)$ & $3(1-4)$ & $3(1-5)$ & $3(1-4)$ & $4(2-5)$ \\
\hline \multicolumn{6}{|l|}{ Microbiological isolates, $N(\%)$} \\
\hline S. aureus & $17(34)$ & $4(33)$ & $4(50)$ & $5(28)$ & $4(33)$ \\
\hline CoNS & $17(34)$ & $2(17)$ & - & $8(44)$ & $7(58)$ \\
\hline Other Gram-positive ${ }^{c}$ & $5(10)$ & $1(8)$ & $1(12)$ & $3(16)$ & - \\
\hline No isolate & $11(22)$ & $5(42)$ & $3(38)$ & $2(12)$ & $1(9)$ \\
\hline Oxacillin-R Staphylococci, $n(\%)$ & $19(38)$ & $2(16)$ & $4(50)$ & $8(44)$ & $5(41)$ \\
\hline Polymicrobial isolates, ${ }^{\mathrm{d}}, n(\%)$ & $9(18)$ & - & $1(11)$ & $5(27)$ & $3(25)$ \\
\hline
\end{tabular}

ABSSSIs acute bacterial skin and skin structure infections, CIED cardiac implantable electronic device, S. aureus Staphylococcus aureus, CoNS coagulase-negative Staphylococci spp., Oxacillin-R Staphylococci oxacillin-resistant Staphylococci

${ }^{a}$ Eight native vertebral infections; 4 long-bones osteomyelitis; 2 prosthetic devices infections; 4 septic arthritis

${ }^{\mathrm{b}}$ Eleven pace maker infections; 1 aortic vascular graft infection

${ }^{\mathrm{c}}$ E. faecalis (3 pts); C. striatum (1 pt); B. cereus $(1 \mathrm{pt})$

${ }^{\mathrm{d}}$ S. aureus + CoNS (2 isolates); different CoNS (2 isolates); Escherichia faecalis + CoNS (2 isolates); CoNS + Streptococcus parasanguinis (1 isolate); S. aureus + Acinetobacter baumannii (1 isolate); E. coli + CoNS (1 isolate) 
Cultures of the collected specimens (blood, samples from ABSSSI, bone biopsy, synovial fluid, CIED leads) were positive in $39(78 \%)$ patients. The most frequent isolates were represented by S. aureus in 17 (34\%) subjects and coagulasenegative Staphylococci (CoNS) in 17 (34\%). Nine subjects (18\%) presented a polymicrobial infection.

\subsection{Antimicrobial Therapy}

Table 3 illustrates both the antimicrobial treatment and the clinical outcome of the study population. A surgical source control, including the removal of infected implants, was associated with antibiotic treatment in $30 \%$ out of the 50 patients overall, in $25 \%$ of those with ABSSSI, in $11 \%$ with bone and osteoarticular infections, and in $66 \%$ with CIED/ vascular graft infections.

After a median time of 14 (7-28) days, the initial antimicrobial therapy was switched to dalbavancin, mainly in order to allow an early discharge of clinically stable patients (44 subjects, $88 \%$ ).

The first dalbavancin dose of $1500 \mathrm{mg}$ was administered on the same day of hospital discharge in 36/50 (72\%) patients, and on a median of 4 (range 1-19) days before dismissal in the remaining 14 (28\%). Patients with infections requiring an antimicrobial therapy longer than 14 days following the dalbavancin switch received additional infusions of dalbavancin $1500 \mathrm{mg}$ repeated every 14 days in an outpatient setting, for a total duration according to the I.D. consultant prescription (see Sect. 2.1).

In our case series, the median number of dalbavancin $1500 \mathrm{mg}$ doses administered per patient was 1 (IQR 1-3), but significant inter-subject differences were observed, with the longest treatment duration prescribed in the group of $\mathrm{CIED/vascular}$ grafts infections, in which up to three subsequent doses (total treatment duration 6 weeks) were administered $(p=0.015)$.

Dalbavancin showed a high rate of clinical success $(98 \%)$ at the EOT, along with great tolerability. Adverse effects were reported only for one patient, who complained of a severe headache, which resulted in the early interruption of the treatment after the first dose of dalbavancin. This subject, nonetheless, showed a clinical success and no relapse throughout a 6-month follow-up.

A median follow-up of 150 (IQR 30-180) days was available for 40 patients (80\%), 37 of whom $(93 \%)$ showed no signs of infection relapse, while a clinical relapse was

Table 3 Antimicrobial treatment and clinical outcome of study population

\begin{tabular}{|c|c|c|c|c|c|}
\hline Variables & Total $(n=50)$ & ABSSSIs $(n=12)$ & $\begin{array}{l}\text { Complicated } \\
\text { ABSSSIs } \\
(n=8)\end{array}$ & $\begin{array}{l}\text { Bone and joints } \\
\text { infections ( } n= \\
18)\end{array}$ & $\begin{array}{l}\text { CIED/vascular } \\
\text { grafts infections } \\
(n=12)\end{array}$ \\
\hline \multicolumn{6}{|l|}{ In-hospital antimicrobial regimens, $n(\%)$} \\
\hline Beta-lactam-based & $3(6)$ & $2(16)$ & $1(12)$ & - & - \\
\hline Glycopeptide-based & $4(8)$ & $2(16)$ & $1(12)$ & $1(5)$ & - \\
\hline Daptomycin & $38(76)$ & $7(60)$ & $5(64)$ & $15(83)$ & $11(19)$ \\
\hline Other combinations $^{\mathrm{a}}$ & $5(10)$ & $1(8)$ & $1(12)$ & $2(12)$ & $1(9)$ \\
\hline Source control, $n(\%)$ & $15(30)$ & $3(25)$ & $2(25)$ & $2(11)$ & $8(66)$ \\
\hline \multicolumn{6}{|l|}{ Indication to switch to dalbavancin, $n(\%)$} \\
\hline Early hospital discharge & $44(88)$ & $11(92)$ & $6(75)$ & $15(83)$ & $12(100)$ \\
\hline Failure of initial therapy & $5(10)$ & $1(8)$ & $2(25)$ & $2(11)$ & - \\
\hline Adverse events to initial therapy & $1(2)$ & - & - & $1(5)$ & - \\
\hline $\begin{array}{l}\text { Dalbavancin doses (1500 mg-dose), median } \\
\text { (IQR) }^{\mathrm{b}}\end{array}$ & $1(1-3)$ & $1(1-1)$ & $2(2-4)$ & $1(1-3)$ & $3(1-3)$ \\
\hline \multicolumn{6}{|l|}{ Outcome of antimicrobial treatment, $n(\%)$} \\
\hline Clinical success & $49(98)$ & $12(100)$ & $8(100)$ & $17(95)$ & $12(100)$ \\
\hline Treatment failure (due to adverse events) & $1(2)$ & - & - & $1(5)$ & - \\
\hline \multicolumn{6}{|l|}{ Outcome at follow-up (FU), $n(\%)$} \\
\hline Days of FU, median (IQR) & $150(30-180)$ & - & - & - & - \\
\hline Relapses, $n(\%)$ & $3(6)$ & $1(8)$ & $1(12)$ & - & $1(8)$ \\
\hline Lost to FU, $n(\%)$ & $10(20)$ & $5(41)$ & $1(12)$ & $3(16)$ & $1(8)$ \\
\hline
\end{tabular}

ABSSSIs acute bacterial skin and skin structure infections, CIED cardiac implantable electronic device, S. aureus Staphylococcus aureus, CoNS coagulase-negative Staphylococci spp., Oxacillin-R isolates oxacillin-resistant isolates

${ }^{\mathrm{a}}$ Gentamicin monotherapy (2 patients), levofloxacin monotherapy (2 patients), clindamycin + meropenem (1 patient)

${ }^{\mathrm{b}}$ Thirty-five patients received dalbavancin as monotherapy and 15 in combination therapy mainly with a beta-lactam or trimethoprim/sulfamethoxazole 
observed in three patients (7\%) 3 months after the EOT in all cases. Two of these relapses were in a 77-year-old woman with a prosthetic hip joint infection (no isolate available) and a 72-year-old man with a CIED infection with methicillinsensitive $S$. epidermidis. In both patients, surgical source control had not been performed due to multiple co-morbidities. The third patient was an intravenous drug user, who presented with a new ABSSSI episode on the right arm. The inability to culture any pathogen at the time of all these three relapses, as well as the intravenous injections of illegal drugs in the third patients, did not allow us to establish with certainty if either a recurrence of the prior infection or a new infection occurred.

\subsection{Hospital Length-of-Stay and Treatment Costs Following the Switch to Dalbavancin Therapy}

Data regarding the duration of antimicrobial therapy and hospital LOS are shown in Table 4. Overall, we observed a median actual LOS of 22 days (IQR 11-33) per patient without any significant difference across the various types of infections. On the other hand, the overall median hypothetical LOS, estimated if no switch to dalbavancin would have occurred, was 47 days (IQR 35-67) per patient. Therefore, the early hospital discharge following the switch to dalbavancin therapy allowed a median reduction in hospital stay of 14 days (IQR 14-49) per patient.

As expected, a significantly different hypothetical LOS was seen among patients according to the site of infections $(p=0.009)$. The greater reduction in LOS following the switch to dalbavancin was observed in those infections requiring longer antimicrobial treatment, i.e., complicated
ABSSSI, osteoarticular and CIED/vascular graft infections, in which a median of 28 (IQR 28-42), 21 (IQR 21-49), and 42 (IQR 14-42) days per patient were saved, respectively ( $p$ $=0.015$ vs. other groups).

Table 5 shows the costs of both the actual treatment with dalbavancin and the hypothetical comparator of standard antimicrobial therapy. Costs saved by switching standard antimicrobials to dalbavancin are also reported.

Overall, the median individual expense of antimicrobial treatment with dalbavancin (including medications, infusion sets, accessories, and hospital accommodation) was $€ 1178$ (IQR 1160-3518). On the other hand, if no switch to dalbavancin would have occurred and patients would have completed the same in-hospital antimicrobial therapy prior to dalbavancin switch, the median individual expense would have increased up to $€ 9660$ (IQR 7135-20,845). A median amount of $€ 8,259$ (IQR 5644-17,270) was hence saved for each patient by switching to dalbavancin therapy, with the greatest saving due to the reduced hospital stay [€7846 (IQR 5502-15,756)].

Notably, the expense reduction in the treatment-related cost following dalbavancin use was significantly different according to the site of infection. The greater financial saving was observed for patients with CIED/vascular graft infections: in this setting, a median of $€ 20,084$ per patient (IQR 8259-24,366; $p=0.007$ ) was saved.

Considering the total of 50 patients enrolled, we estimated that 1358 patient-days of hospital-stay and $€ 998,368$ were saved overall following the use of the long-acting antimicrobial. The greatest cost saving ( $€ 953,103)$ was ascribed to hospital accommodation.

Table 4 Actual and hypothetical duration of antimicrobial therapy and hospital length-of-stay (LOS) in 50 patients with different Gram-positive infections.

\begin{tabular}{|c|c|c|c|c|c|c|}
\hline Variables & Total $(n=50)$ & ABSSSIs $(n=12)$ & $\begin{array}{l}\text { Complicated } \\
\text { ABSSSIs } \\
(n=8)\end{array}$ & $\begin{array}{l}\text { Bone and joints } \\
\text { infections }(n=18)\end{array}$ & $\begin{array}{l}\text { CIED/vascular grafts } \\
\text { infections }(n=12)\end{array}$ & $p$ value \\
\hline \multicolumn{7}{|c|}{ Duration of antimicrobial therapy [days/patient, median (IQR)] } \\
\hline Prior to dalbavancin therapy & $10(4-23)$ & $2(2-4)$ & $12(5-32)$ & $18(16-32)$ & $13(8-28)$ & $<0.001$ \\
\hline After switch to dalbavancin ${ }^{\mathrm{a}}$ & $14(14-42)$ & $14(14-14)$ & $28(28-56)$ & $14(14-42)$ & $42(14-42)$ & 0.033 \\
\hline \multicolumn{7}{|c|}{ Hospital LOS [days/patient, median (IQR)] ${ }^{\mathrm{b}}$} \\
\hline Actual & $22(11-33)$ & $13(10-23)$ & $12(6-25)$ & $28(19-38)$ & $21(14-33)$ & 0.053 \\
\hline Hypothetical $^{\mathrm{c}}$ & $47(35-67)$ & $30(24-42)$ & $58(41-68)$ & $50(40-74)$ & $55(35-76)$ & 0.009 \\
\hline Reduction in hospital LOS & $14(14-49)$ & $14(14-14)$ & $28(28-42)$ & $21(21-49)$ & $42(14-42)$ & 0.015 \\
\hline
\end{tabular}

ABSSSIs acute bacterial skin and skin structure infections, CIED cardiac implantable electronic device, IQR interquartile range

${ }^{a}$ End of dalbavancin therapy has been considered to be at 14 days after the last $1500 \mathrm{mg}$ dalbavancin dose (see text, Sect. 2.1 "Definitions" paragraph)

${ }^{\mathrm{b}}$ Overall length of stay since hospital admission, which included the time period prior to start of antimicrobial therapy and was possibly influenced by other clinical problems other than the infection

${ }^{\mathrm{c}}$ Hypothetical duration of hospitalization was estimated considering that the initial standard antimicrobial treatment would have been administered in hospital, without switching to dalbavancin, until the end of therapy (see text, "Methods" Sect. 2) 
Table 5 Expense for both actual treatment with dalbavancin and hypothetical continuation of in-hospital standard treatment

\begin{tabular}{|c|c|c|c|c|c|c|}
\hline Variables & Total $(n=50)$ & ABSSSIs $(n=12)$ & $\begin{array}{l}\text { Complicated ABSS- } \\
\text { SIs }(n=8)\end{array}$ & $\begin{array}{l}\text { Bone and joints } \\
\text { infections }(n=18)\end{array}$ & $\begin{array}{l}\text { CIED/vascular grafts } \\
\text { infections }(n=12)\end{array}$ & $p$ value \\
\hline \multicolumn{7}{|c|}{ Actual dalbavancin treatment } \\
\hline Antimicrobials & $1160(1160-3481)$ & $1160(1160-1160)$ & $2707(2320-4641)$ & $1160(1160-3481)$ & $3481(1160-3481)$ & 0.017 \\
\hline $\begin{array}{l}\text { Infusion sets and } \\
\text { accessories }\end{array}$ & $0(0-37)$ & - & $37(18-37)$ & $37(0-37)$ & $37(0-37)$ & 0.018 \\
\hline $\begin{array}{l}\text { Hospital } \\
\text { accommodation }^{\mathrm{a}}\end{array}$ & $0(0-375)$ & $164(0-1735)$ & $0(0-374)$ & $0(0-374)$ & - & 0.109 \\
\hline $\begin{array}{l}\text { Total cost of dalba- } \\
\text { vancin treatment }\end{array}$ & $1178(1160-3518)$ & $1160(1160-1160)$ & $2744(2339-4679)$ & $1197(1160-3518)$ & $3518(1160-3518)$ & 0.013 \\
\hline \multicolumn{7}{|c|}{ Hypothetical continuation of in-hospital standard treatment } \\
\hline Antimicrobials & $1690(1642-5019)$ & $1545(287-1673)$ & $5302(3346-6692)$ & $4296(1673-4973)$ & $1673(1642-5019)$ & 0.009 \\
\hline $\begin{array}{l}\text { Infusion sets and } \\
\text { accessories }\end{array}$ & $23(19-56)$ & $19(18-19)$ & $43(36-73)$ & $31(19-57)$ & $55(18-57)$ & 0.031 \\
\hline $\begin{array}{l}\text { Hospital accommo- } \\
\text { dation }\end{array}$ & $8596(5502-16,506)$ & $5502(5117-7469)$ & $\begin{array}{l}11,642(10,108- \\
13,216)\end{array}$ & $6629(5502-16,506)$ & $\begin{array}{c}18,963(7742 \\
-23,226)\end{array}$ & 0.001 \\
\hline $\begin{array}{l}\text { Total cost of hypo- } \\
\text { thetical continu- } \\
\text { ation of standard } \\
\text { treatment }\end{array}$ & $9660(7135-20,845)$ & $6765(5784-8346)$ & $\begin{array}{l}16,094(14,328- \\
19,925)\end{array}$ & $8328(7149-21,538)$ & $\begin{array}{l}23,603(9419- \\
27,884)\end{array}$ & 0.002 \\
\hline \multicolumn{7}{|c|}{ Costs saved by switching to dalbavancin } \\
\hline Antimicrobials & $530(409-1537)$ & $385(-872$ to -530$)$ & $2050(1025-2076)$ & $512(482-1288)$ & $1144(512-1537)$ & 0.005 \\
\hline $\begin{array}{l}\text { Infusion sets and } \\
\text { accessories }\end{array}$ & $19(18-20)$ & $18(18-19)$ & $27(4.56-35)$ & $19(17-21)$ & $18(18-20)$ & 0.951 \\
\hline $\begin{array}{l}\text { Hospital accommo- } \\
\text { dation }\end{array}$ & $7846(5502-15,756)$ & 5383 (3129-6719) & $\begin{array}{l}11,156(10,108- \\
13,216)\end{array}$ & $6629(5502-15,756)$ & $\begin{array}{l}18,963(7742- \\
23,226)\end{array}$ & 0.001 \\
\hline Total cost saved & $8259(5644-17,270)$ & $5034(3647-6590)$ & $\begin{array}{l}12,882(11,970- \\
15,246)\end{array}$ & $7131(5644-17,270)$ & $\begin{array}{l}20,084(8259- \\
24,366)\end{array}$ & 0.007 \\
\hline
\end{tabular}

Values are expressed as $€$ per patient, median (IQR)

ABSSSIs acute bacterial skin and skin structure infections, CIED cardiac implantable electronic device, IQR interquartile range

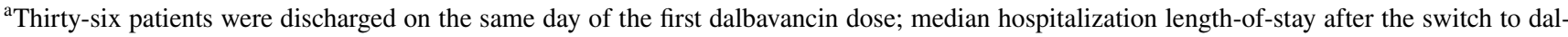
bavancin in the remaining 14 subjects was 2 days (range 1-11)

\section{Discussion}

Gram-positive bacteria are one of the most common causes of bacterial infections in humans, both community and hospital acquired, with a very wide spectrum of clinical presentations, a significant burden of morbidity and mortality, and a huge impact on the capacity and expense of the National Healthcare Systems [23].

The management of Gram-positive-related osteoarticular and cardiovascular infections, including implant-associated infections, can be considered one of the most challenging for I.D. specialists, especially when multidrug-resistant strains are involved $[24,25]$. These infections require prolonged and complex antimicrobial treatments, generally combined with source-control procedures [26-28]. Moreover, even noncomplicated Gram-positive infections with a low mortality rate (e.g., ABSSSIs) represent a relevant concern due to their high prevalence in community settings along with the high percentage of recurrences and hospital re-admissions involved [29].

In this study, we described the real-life clinical outcome as well the impact of dalbavancin therapy on the hospital LOS and on treatment-related costs in a wide spectrum of Gram-positive-related infections, i.e., ABSSSIs (non-complicated and complicated), osteoarticular and prosthetic joint infections, CIED and vascular graft infections, in patients hospitalized in different specialty hospital units, both medical and surgical.

In our experience, dalbavancin demonstrated great efficacy and tolerability, in line with previous studies [13, 19, $28,30]$, allowing a high rate of clinical recovery both at the EOT and at follow-up visits, along with a very low incidence of adverse effects.

Remarkably, the use of the long-acting antimicrobial dalbavancin was associated with a significant saving both in the hospital LOS and in the antimicrobial treatment-related costs in all infection sites observed. Both these savings were 
greater in the contexts of complex infections such as osteoarticular and cardiovascular infections, which require a longer antimicrobial therapy.

It is worth mentioning that in many cases a relevant part of the hospital LOS occurred before the initiation of antimicrobial therapy: this was related to multiple factors including co-morbidities, diagnostic work-up, and surgical interventions. In particular, ABSSSIs rarely represented the cause of hospitalization in our study groups with this infection site, but rather occurred as an in-hospital complication. This is the reason for the prolonged median LOS of patients in the ABSSSI group. However, this issue did not influence the estimation of LOS reduction following the therapeutic switch to dalbavancin, since the hospital stay before dalbavancin initiation was identical in the hypothetical control of standard treatment (Fig. 1).

In our experience, repeated doses of $1500 \mathrm{mg}$ of dalbavancin were administered every 14 days in patients requiring more than a single dose of this drug. Nevertheless, recent studies observed that lower doses of consolidation treatment with dalbavancin (e.g., $1000 \mathrm{mg}$ every 14 days following the first dose of $1500 \mathrm{mg}$ ) could be used in these complex infections [31-37]. Furthermore, some authors have proposed only two $1500 \mathrm{mg}$ doses of dalbavancin (on days 1 and 8, respectively) as an effective treatment of osteomyelitis and complicated bacteremia or endocarditis due to Gram-positive bacteria [14]. Both these strategies could optimize the use of dalbavancin and allow an even greater cost-saving than that observed in our study population.

Our data suggest that an "early discharge" strategy based on the use of long-acting antimicrobials such as dalbavancin could represent an efficient and advantageous way to manage complex, multidrug-resistant Gram-positive-related infections in order to shorten the patient hospital LOS, thus increasing the availability of hospital beds and saving costs, at least for those patients not eligible for oral antibiotic therapies and who cannot access daily outpatient parenteral antibiotic therapy. Moreover, therapeutic approaches that allow an early patient discharge, like that investigated here, are especially useful in special circumstances such as the current SARS-CoV-2 pandemic, in which the shortage of available hospital beds is critical for patients affected by both COVID-19 and conventional diseases

In addition, other relevant, potential benefits of longacting intravenous antimicrobials warrant consideration and investigation, such as: (a) guaranteed patient adherence to treatment; (b) no need for either a daily inserted or longlasting vascular access, and the consequent decrease of intravenous catheter-associated complications, both infectious and non-infectious [5]; (c) improved patient quality of life due to both the early hospital discharge and the avoidance of daily antimicrobial infusions [6]; (d) decreased healthcare associated infections.

\subsection{Study Limitations}

Our study has the following limitations:

(a) The relatively small number of subjects enrolled, which implies that evaluation of our results on a wider cohort of patients is warranted;

(b) The retrospective, though real-life, design, in which the antimicrobial therapy and hospital LOS depended on the specific clinical situation and on the clinician's judgment rather than on a randomization process;

(c) The choice of an intra-subject control of the observed switch to dalbavancin therapy, which was compared to a hypothetical treatment scenario of in-hospital continuation of the initial standard antimicrobial therapy for the same duration as the actual dalbavancin treatment. We have addressed the reasons that prompted us to choose this study design in the Introduction (Sect. 1). However, we cannot conclude that our results would be confirmed in a study with a real-life matched control group treated in hospital with standard intravenous antimicrobials.

In fact, in our study design the duration of the hypothetical comparator standard treatment, as well as the consequent length of hospitalization and treatment costs, could have been partly either overestimated (in particular for less severe infections such as ABSSSIs), or underestimated (e.g., in case of treatment failure, slower response, complications, or adverse effects). In particular, both vancomycin and teicoplanin are associated with a greater incidence of nephrotoxicity than dalbavancin. Moreover, ABSSSIs are known to often be associated with relapses and hospital readmissions [29]. On the other hand, we believe that our intra-subject control approach could have minimized the bias possibly determined by the many patient-related confounders of an external control group, including co-morbidities, infection severity, hospital-course complications, pathogens involved and their antimicrobial susceptibility, effects of previous antimicrobial treatment administered, adverse events, and, most important, the inter-patient variability of antibiotic pharmacokinetic/pharmacodynamic properties.

Furthermore, our study results, which are based on an intra-subject control of hypothetical in-hospital prolongation of standard antibiotic therapy, cannot be extended to those geographic areas where an efficient, well-organized OPAT program is operative, thus allowing an early hospital discharge for patients who need daily administration of standard intravenous antimicrobials.

We chose our hypothetical study control approach since, as we reported in the Introduction (Sect. 1), our local healthcare system, as well as many other geographic areas in Italy and elsewhere, is not yet properly organized with an effective 
and easily accessible OPAT protocol, and, therefore, a prolonged hospitalization is frequently required in order to complete treatments based on hospital-restricted parenteral antimicrobials.

On the other hand, other published studies also adopted our study control approach. Bouza et al. [20] estimated the saving of both hospital LOS and treatment-related costs in different infections treated with dalbavancin as compared with a hypothetical in-hospital therapy with daptomycin for an equivalent length of time to that with dalbavancin. As opposed to Bouza et al. we found it to be more appropriate to not choose as a hypothetical in-hospital control therapy a single comparator molecule for all patients, but rather the actual antimicrobial therapy administered to each patient before dalbavancin. Several other methodological differences characterize the same remarkable article from Bouza et al. as compared with the present study, including the estimation of the daily cost of hospital accommodation (a single standard reference cost calculated nationwide for an internal medicine ward in the Bouza et al. article vs. the actual cost calculated for each participating unit in our study).

(d) Finally, this study was performed under the Italian model of healthcare delivery. Therefore, the expenses calculated in our experience might not be the same in other countries where drug acquisition cost and hospitalization costs are likely to differ. This issue could represent a further topic of investigation.

Notwithstanding the limitations mentioned above, we believe our study has several strengths and originality with respect to the published literature, which have been addressed in the Introduction (Sect. 1).

\section{Conclusions}

Our study suggests that an "early discharge" strategy based on the use of dalbavancin provides a significant reduction in both hospital LOS and treatment-related costs, combined with adequate clinical success and good tolerability. These organizational and economic benefits are particularly appreciable in patients with difficult Gram-positive infections requiring longer antimicrobial treatment, who are noneligible for oral antibiotic therapies and for whom a daily OPAT is not feasible.

\section{Declarations}

Funding No funding was received to conduct this study. Open access funding provided by Universitã di Foggia within the CRUI-CARE Agreement.
Conflict of interest No authors have any relevant financial, personal, political, intellectual, or religious interests that might affect the objectiveness of the presented data.

Ethics approval This research study was conducted retrospectively from data obtained for clinical purposes. We consulted extensively with the Ethical Committee of Hospital Policlinico of Bari who determined that our study did not need ethical approval according to Italian law. In any case, data for all patients were recorded anonymously and managed following the Italian requirements regarding the privacy protections and in accordance with the 1964 Helsinki Declaration (and its amendments).

Consent to participate When dalbavancin or other antimicrobials were prescribed for indications different to those approved by the European Medicines Agency, patients provided informed written consent before their use. All patients provided informed consent for the use of their data for research purposes. Furthermore, data were previously anonymized according to the requirements set out by the Italian Data Protection Code (leg. Decree 196/2003).

Consent to publish Not applicable.

Availability of data and materials All data generated or analyzed during this study are included in the published article.

Code availability Not applicable.

Authors' contributions All authors contributed to the study conception and design, in particular Sergio Carbonara, who conceived the study design and performed a supervisory role throughout the duration of the project, providing fundamental support in writing and revising the manuscript presented for publication. Material preparation, data collection, and analysis were performed by Mariacristina Poliseno, Davide Fiore Bavaro, and Camilla Taglietti. The first draft of the manuscript was written by Mariacristina Poliseno and all authors commented on previous versions of the manuscript. All authors read and approved the final manuscript.

Open Access This article is licensed under a Creative Commons Attribution-NonCommercial 4.0 International License, which permits any non-commercial use, sharing, adaptation, distribution and reproduction in any medium or format, as long as you give appropriate credit to the original author(s) and the source, provide a link to the Creative Commons licence, and indicate if changes were made. The images or other third party material in this article are included in the article's Creative Commons licence, unless indicated otherwise in a credit line to the material. If material is not included in the article's Creative Commons licence and your intended use is not permitted by statutory regulation or exceeds the permitted use, you will need to obtain permission directly from the copyright holder. To view a copy of this licence, visit http://creativecommons.org/licenses/by-nc/4.0/.

\section{References}

1. European Centre for Disease Prevention and Control. Point prevalence survey of healthcare-associated infections and antimicrobial use in European acute care hospitals, 2011-2012. Stockholm: ECDC; 2013. http://www.ecdc.europa.eu/en/publications/Publi cations/healthcare-associated-infectionsantimicrobial-use-PPS. pdf. Accessed 12 Jan 2021.

2. Seng P, Amrane S, Million M, Stein A. Old antimicrobials and Gram-positive cocci through the example of infective endocarditis 
and bone and joint infections. Int J Antimicrob Agents. 2017. https://doi.org/10.1016/j.ijantimicag.2017.03.004.

3. Revest M, Camou F, Senneville E, et al. Medical treatment of prosthetic vascular graft infections: review of the literature and proposals of a Working Group. Int J Antimicrob Agents. 2015. https://doi.org/10.1016/j.ijantimicag.2015.04.014.

4. Pavoni GL, Giannella M, Falcone M, et al. Conservative medical therapy of prosthetic joint infections: retrospective analysis of an 8-year experience. Clin Microbiol Infect. 2004. https://doi.org/10. 1111/j.1469-0691.2004.00928.x.

5. Keller SC, Williams D, Gavgani M, Hirsch D, Adamovich J, Hohl D, Krosche A, Cosgrove S, Perl TM. Environmental exposures and the risk of central venous catheter complications and readmissions in home infusion therapy patients. Infect Control Hosp Epidemiol. 2017;38(1):68-75. https://doi.org/10.1017/ice.2016. 223

6. Keller SC, Williams D, Levering M, Cosgrove SE. Health-related quality of life in outpatient parenteral antimicrobial therapy. Open Forum Infect Dis. 2018;5(7):143. https://doi.org/10.1093/ofid/ ofy 143.

7. Dash RP, Babu RJ, Srinivas NR. Review of the pharmacokinetics of DBV, a recently approved lipoglycopepatientide antibiotic. Infect Dis (Lond). 2017. https://doi.org/10.1080/23744235.2017. 1296968.

8. Fernández J, Greenwood-Quaintance KE, Patel R. In vitro activity of DBV against biofilms of staphylococci isolated from prosthetic joint infections. Diagn Microbiol Infect Dis. 2016. https://doi.org/ 10.1016/j.diagmicrobio.2016.05.009.

9. Darouiche RO, Mansouri MD. DBV compared with vancomycin for prevention of Staphylococcus aureus colonization of devices in vivo. J Infect. 2005. https://doi.org/10.1016/j.jinf.2004.05.006.

10. Scoble PJ, Owens RC Jr, Puttagunta S, et al. Pharmacokinetics, safety, and tolerability of a single 500-mg or 1000-mg intravenous dose of DBV in healthy Japanese subjects. Clin Drug Investig. 2015. https://doi.org/10.1007/s40261-015-0340-4.

11. Dunne MW, Talbot GH, Boucher HW, et al. Safety of DBV in the treatment of skin and skin structure infections: a pooled analysis of randomized, comparative studies. Drug Saf. 2016. https://doi. org/10.1007/s40264-015-0374-9.

12. Soriano A, Rossolini GM, Pea F. The role of DBV in the treatment of acute bacterial skin and skin structure infections (ABSSSIs). Expert Rev Anti Infect Ther. 2020. https://doi.org/10.1080/14787 210.2020.1746643.

13. Bassetti M, Magnasco L, Del Puente F, Giacobbe DR. Role of new antibiotics in the treatment of acute bacterial skin and skinstructure infections. Curr Opin Infect Dis. 2020. https://doi.org/ 10.1097/QCO.0000000000000631.

14. Rappo U, Puttagunta S, Shevchenko V, et al. Dalbavancin for the treatment of osteomyelitis in adult patients: a randomized clinical trial of efficacy and safety. Open Forum Infect Dis. 2018;6(1):331. https://doi.org/10.1093/ofid/ofy331.

15. Morata L, Cobo J, Fernández-Sampedro M, et al. Safety and efficacy of prolonged use of dalbavancin in bone and joint infections. Antimicrob Agents Chemother. 2019. https://doi.org/10.1128/ AAC.02280-18

16. Bai F, Aldieri C, Cattelan A, et al. Efficacy and safety of dalbavancin in the treatment of acute bacterial skin and skin structure infections (ABSSSIs) and other infections in a real-life setting: data from an Italian observational multicentric study (DALBITA study). Expert Rev Anti Infect Ther. 2020. https://doi.org/10.1080/ 14787210.2020.1798227.

17. Agarwal R, Bartsch SM, Kelly BJ, et al. Newer glycopeptide antibiotics for treatment of complicated skin and soft tissue infections: systematic review, network meta-analysis and cost analysis. Clin Microbiol Infect. 2018. https://doi.org/10.1016/j.cmi.2017.08.028.
18. Turco NJ, Kane-Gill SL, Hernandez I. A cost-minimization analysis of dalbavancin compared to conventional therapy for the outpatient treatment of acute bacterial skin and skin-structure infections. Expert Opin Pharmacother. 2018. https://doi.org/10.1080/ 14656566.2018.1442439.

19. Streifel AC, Sikka MK, Bowen CD, Lewis JS 2nd. Dalbavancin use in an academic medical centre and associated cost savings. Int J Antimicrob Agents. 2019. https://doi.org/10.1016/j.ijantimicag. 2019.08.007.

20. Bouza E, Valerio M, Soriano A, Morata L, et al. Dalbavancin in the treatment of different Gram-positive infections: a real-life experience. Int J Antimicrob Agents. 2018. https://doi.org/10. 1016/j.ijantimicag.2017.11.008.

21. Marcellusi A, Bini C, Andreoni MS, et al. Expense impact analysis of dalbavancin in the treatment of acute bacterial skin and skin structure infections in Three European Countries. Clin Drug Investig. 2020. https://doi.org/10.1007/s40261-020-00891-w.

22. Arrieta-Loitegui M, Caro-Teller JM, Ortiz-Pérez S, et al. Effectiveness, safety and cost analysis of dalbavancin in clinical practice. Eur J Hosp Pharm. 2020. https://doi.org/10.1136/ejhph arm-2020-002315.

23. US Food and Drug Administration. Acute bacterial skin and skin structure infections: developing drugs for treatment. Guidance for industry. 2013. https://www.fda.gov/downloads/Drugs/Guidances/ ucm071185.pdf. Accessed 12 Jan 2021.

24. De Kraker ME, Wolkewitz M, Davey PG, et al. Clinical impact of antimicrobial resistance in European hospitals: excess mortality and length of hospital stay related to methicillin-resistant Staphylococcus aureus bloodstream infections [published correction appears in Antimicrob Agents Chemother]. Antimicrob Agents Chemother. 2011. https://doi.org/10.1128/AAC.01157-10.

25. Lee AS, de Lencastre H, Garau J, et al. Methicillin-resistant Staphylococcus aureus. Nat Rev Dis Prim. 2018. https://doi.org/ 10.1038/nrdp.2018.33.

26. Haessler S, Mackenzie T, Kirkland KB. Long-term outcomes following infection with meticillin-resistant or meticillin-susceptible Staphylococcus aureus. J Hosp Infect. 2008. https://doi.org/10. 1016/j.jhin.2008.01.008.

27. Galar A, Weil AA, Dudzinski DM, et al. Methicillin-resistant Staphylococcus aureus prosthetic valve endocarditis: pathophysiology, epidemiology, clinical presentation, diagnosis, and management. Clin Microbiol Rev. 2019. https://doi.org/10.1128/ CMR.00041-18.

28. Davis JS, Van Hal S, Tong SY. Combination antibiotic treatment of serious methicillin-resistant Staphylococcus aureus infections. Semin Respir Crit Care Med. 2015;36(1):3-16. https://doi.org/10. 1055/s-0034-1396906.

29. Kaye KS, Petty LA, Shorr AF, Zilberberg MD. Current epidemiology, etiology, and burden of acute skin infections in the United States. Clin Infect Dis. 2019;68(Suppl 3):S193-9. https://doi.org/ 10.1093/cid/ciz002.

30. Koziatek C, Mohan S, Caspers C, et al. Experience with dalbavancin for cellulitis in the emergency department and emergency observation unit. Am J Emerg Med. 2018. https://doi.org/10. 1016/j.ajem.2017.11.037.

31. Guest JF, Esteban J, Manganelli AG, et al. Comparative efficacy and safety of antibiotics used to treat acute bacterial skin and skin structure infections: results of a network meta-analysis. PLoS ONE. 2017. https://doi.org/10.1371/journal.pone.0187792.

32. Cahill TJ, Baddour LM, Habib G, et al. Challenges in infective endocarditis. J Am Coll Cardiol. 2017. https://doi.org/10.1016/j. jacc.2016.10.066.

33. Raad I, Darouiche R, Vazquez J, et al. Efficacy and safety of weekly dalbavancin therapy for catheter-related bloodstream infection caused by Gram-positive pathogens. Clin Infect Dis. 2005;40(3):374-80. 
34. Soriano A, Rossolini GM, Pea F. The role of Dalbavancin in the treatment of acute bacterial skin and skin structure infections (ABSSSIs). Expert Rev Anti Infect Ther. 2020. https://doi.org/ 10.1080/14787210.2020.1746643.

35. Dunne MW, Puttagunta S, Giordano P, et al. Randomized clinical trial of single-dose versus weekly dalbavancin for treatment of acute bacterial skin and skin structure infection. Clin Infect Dis. 2016;62(5):545-51. https://doi.org/10.1093/cid/civ982.
36. Baldoni D, Furustrand Tafin U, Aeppli S, et al. Activity of dalbavancin, alone and in combination with rifampicin, against meticillin-resistant Staphylococcus aureus in a foreign-body infection model. Int J Antimicrob Agents. 2013. https://doi.org/10.1016/j. ijantimicag.2013.05.019.

37. Bassetti M, Peghin M, Carnelutti A, Righi E. The role of dalbavancin in skin and soft tissue infections. Curr Opin Infect Dis. 2018. https://doi.org/10.1097/QCO.0000000000000430.

\section{Authors and Affiliations}

\section{Mariacristina Poliseno ${ }^{1,9} \oplus$. Davide Fiore Bavaro ${ }^{1} \cdot$ Gaetano Brindicci $^{1}$. Giovanni Luzzi ${ }^{2} \cdot$ Domenico Maria Carretta $^{3}$. Antonio Spinarelli ${ }^{4} \cdot$ Raffaella Messina ${ }^{5} \cdot$ Maria Paola Miolla ${ }^{4} \cdot$ Teresa Immacolata Achille ${ }^{2}$. Maria Rosaria Dibartolomeo ${ }^{6}$. Maria Dell'Aera ${ }^{6}$. Annalisa Saracino ${ }^{1}$. Gioacchino Angarano ${ }^{1}$. Stefano Favale ${ }^{2}$. Carlo D'Agostino $^{3} \cdot$ Biagio Moretti $^{4} \cdot$ Francesco Signorelli $^{5} \cdot$ Camilla Taglietti $^{7} \cdot$ Sergio Carbonara ${ }^{1,8}$}

1 Department of Biomedical Sciences and Human Oncology, Clinic of Infectious Diseases, University of Bari, Bari, Italy

2 Unit of Cardiovascular Diseases, Department of Emergency and Organ Transplantation, University of Bari, Bari, Italy

3 Cardiology Department, University, Hospital Policlinico Consorziale, Bari, Italy

4 Orthopaedics Unit, Department of Basic Medical Science, Neuroscience and Sensory Organs, University of Bari, Bari, Italy
5 Division of Neurosurgery, Department of Basic Medical Sciences, Neurosciences and Sense Organs, University of Bari, Bari, Italy

6 Department of Hospital Pharmacy, Policlinico of Bari, University of Bari, Bari, Italy

7 OPT Srl-Consulenza di Direzione, Milan, Italy

8 Unit of Infectious Diseases, Hospital V. Emanuele II, Bisceglie, Italy

9 Unit of Infectious Diseases, A.O.U. Policlinico Riuniti, Foggia, Italy 